\title{
Description of Plasma Concentrations of Free Amino Acids with Some Clinical Symptoms of Patients Under 5 with Short Bowel Syndrome
}

\author{
Luu Thi My Thuc* and Nguyen Thi Hang \\ Vietnam National Children's Hospital, Vietnam \\ *Corresponding author: Luu Thi My Thuc, Vietnam National Children's Hospital, Vietnam
}

Received: 䟧 February 11, 2019

Published: 㙕 February 20, 2019

\begin{abstract}
Amino acid (AA) is the main component of proteins. Changes in levels of plasma AA concentration have been mentioned in some cases such as plasma AA concentrations in chronic kidney disease (CKD) patients are low when inflammation status increases [1] or patients with short bowel syndrome (SBS) undergoing total parenteral nutrition (TPN) have decreasing concentrations of essential amino acids (EAA), increasing rate of malnutrition and complications while for those undergoing part of parenteral nutrition (PN), there was just a decrease in concentrations of two branched chain amino acids, leucin, valine, cysteine, and tyrosine [2]. Autistic children had significant lower plasma levels of leucine, isoleucine, phenylalanine, methionine, cysteine, serine, tyrosine while phosphoserine was significantly raised [3]. In SBS, as intestinal absorption of nutrients is significantly decreased, patients are largely reliant on PN so the concentrations and compositions of AA in PN formula is critical to treatment outcomes. The study was carried out with the

Objective: describe the plasma concentrations of free amino acids in 57 children with SBS and the correlation with clinical symptoms. Method: descriptive cross-sectional study.

Result: High ratio of malnutrition with $96.5 \%$ of underweight, $64.9 \%$ of stunting and $93 \%$ of wasting. All children with SBS had increase of EAA and decrease of non-essential amino acids (NEAA), 38.9\% had histidine deficiency, 19.2\% had tryptophan deficiency, $14 \%$ had arginine deficiency and $12.3 \%$ had isoleucine deficiency. AA deficiency is more common in acquired than in functional SBS, in adaptation than in maintenance phase.
\end{abstract}

Conclusion: High ratio of malnutrition in SBS patients. All children with SBS underwent NEAA deficiencies. Plasma concentrations of free AA largely depended on AA compositions of PN solution.

Keywords: Amino Acid; Short Bowel Syndrome; Children Under 5

\section{Introduction}

AA is mostly absorbed at small intestine; each AA has its own catabolic pathway as well as their metabolites have difference cellular functions. Recently, two AAs that are paid most attention in SBS are glutamine and arginine as they are functional amino acids (FAA) [4]. FAA deficiencies may lead to decrease of protein synthesis, intracellular balance, and metabolism. Therefore, supplementation of specific AA (such as glutamine or arginine) into AA-sufficient diets can still optimize the growth in young animals and prevent diseases (such as obesity, intra-uterine growth restriction) [5].
The question is how the FAA requirement in children with SBS? Currently, there is still little information of FAA requirements. For SBS patients, AA are necessary for physical and mental development, immunity improvement. However, there has not been data for the requirement and amount of $\mathrm{AA}$ in diet for immunity improvement. In most hospitals, besides anthropometric measurements; indices of albumin, protein in plasma are frequently used for nutritional status assessment. AA in plasma are rarely used for nutritional status as well as to follow disease progress, prognosis, treatment outcome especially in children with SBS undergoing TPN. To timely 
and sufficiently provide nutrients to minimize deficiencies and improve immunity for children with SBS through AA in PN solution, this study is carried out to describe the plasma concentrations of free AA with the clinical status of SBS patients.

\section{Subjects and Methods}

The descriptive cross-sectional study was carried out on 57 children under 60 months of age at Vietnam National Children's Hospital from August 2017 to August 2018 with SBS diagnosis, who have post-operation time more than 3 weeks. In which: 15 patients who were not reliant on PN were orally fed (maintenance period), 42 patients were reliant on PN and the PN solution provided 30 - 70\% total energy requirement (adaptation period). Patients in the study did not have diseases or congenital disorders that require long-term $\mathrm{PN}$ or using medications that affect amino acid metabolism. The children were divided into two groups:

a) Acquired SBS: length of the remaining small intestine (bowel) is less than $50 \mathrm{~cm}$ at neonatal stage with gestational age under 36 weeks or less than $72 \mathrm{~cm}$ for full-term babies. If the resection is carried out beyond the infancy stage, length of the remaining intestine is less than $75 \mathrm{~cm}$ in children under 12 months and less than $100 \mathrm{~cm}$ in children over 12 months.

b) Functional SBS: after the resection, children do not meet these above criteria but must be reliant on PN in at least 42 days post-operation. PN solution for intravenous infusion is designed by Pharmacy Department of Vietnam National Children's Hospital. The protein solution is usually vaminolac $6.5 \%$ or amino plasma $10 \%$. The compositions also include sugar, salt, lipid, potassium, vitamins and minerals.

\section{Data Collection Method}

Variables were collected based on the designed medical records to collect clinical symptoms, nutritional status based on WHO guideline (2006) with classifications of underweight (weight/ageW/A), stunting (Height/age-H/A), wasting (Weight/Height-W/H) based on the cut off below -2SD vs. the control group. The children were examined by medical doctors. Only patients in maintenance and adaptation phases were chosen (post-operative time over than 3 weeks) because for the acute phase (under 2 weeks postoperation), patients are usually kept in rehabilitation with intensive care and life-threatening conditions. AA is quantified by high performance solution chromatography at the hospital's testing center, with control from normal children at the same ages. Blood samples were taken, samples from patients in maintenance e phase were taken at least 1 month since the last PN after discharge and from patients who can orally take at least $70 \%$ of energy demand by age. For patients in adaptation phase, blood samples were taken after 4 weeks of PN. With patients that evidences of acquired SBS are insufficient (with a doubt of functional SBS), blood samples were taken similarly with a consecutive following of patients, only when patients underwent PN in more than 42 days, they were listed for the study and the study time was the time of taking samples. The results for AA concentrations of each patient were compared with the normal level of children at the same ages to have the conclusions of increasing, decreasing or normal plasma concentrations of free AA.

\section{Data Analysis}

Data was input and analyzed by the software Statistical Package for Social Sciences (SPSS16.0). Anthropometric data was treated by the software Anthro of WHO 2006.Compare percentage by fisher exact test, compare medium values of 2 groups by non-parametric Mann-Whitney test with $\mathrm{p}<0,05$ as significant. The result (Table 1) showed that SBS was common in children under 6 months of age $(73.7 \%)$ as they had resection at neonatal period (87.7\%). $54.4 \%$ patients had acquired SBS while $46.6 \%$ had functional SBS. $71.9 \%$ had artificial anal sphincter, $73.7 \%$ were in adaptation phase and $26.3 \%$ were in maintenance phase. The result (Graph 1) showed high rate of malnutrition with $96.5 \%$ of underweight, $64.9 \%$ of stunting, $93 \%$ of wasting and most cases are severe malnutrition. The result (Table 2) showed that no patient has leucine and lysine (EAA) deficiency; no patient had asparagine, glutamate (NEAA) deficiency. With EAA, the deficiency rate was highest with histidine (38.6\%), then tryptophan (19.3\%). With NEAA, the deficiency rate was highest with cysteine (19.3\%), serine and glycine (both 15.8\%), then arginine (14\%). All patients had increase in EAA and the most was threonine $(22.8 \%)$, lysine (15.79\%), isoleucine $(12.3 \%)$ and leucine $(10.5 \%)$. With NEAA, no patient had increase with arginine and tyrosine. Glutamate had the highest increase rate $(28.1 \%)$, then asparagine (21.1\%) and glutamine (17.5\%).

Table 1: General characteristics of children with SBS $(n=57)$.

\begin{tabular}{|c|c|c|}
\hline Characteristic & $\mathbf{n}$ & \% \\
\hline Functional SBS & 26 & 45.6 \\
\hline Acquired SBS & 31 & 54.4 \\
\hline Artificial anal sphincter & 41 & 71.9 \\
\hline Adaptation phase & Disease phase & 73.7 \\
\hline Maintenance phase & 42 & 26.3 \\
\hline
\end{tabular}




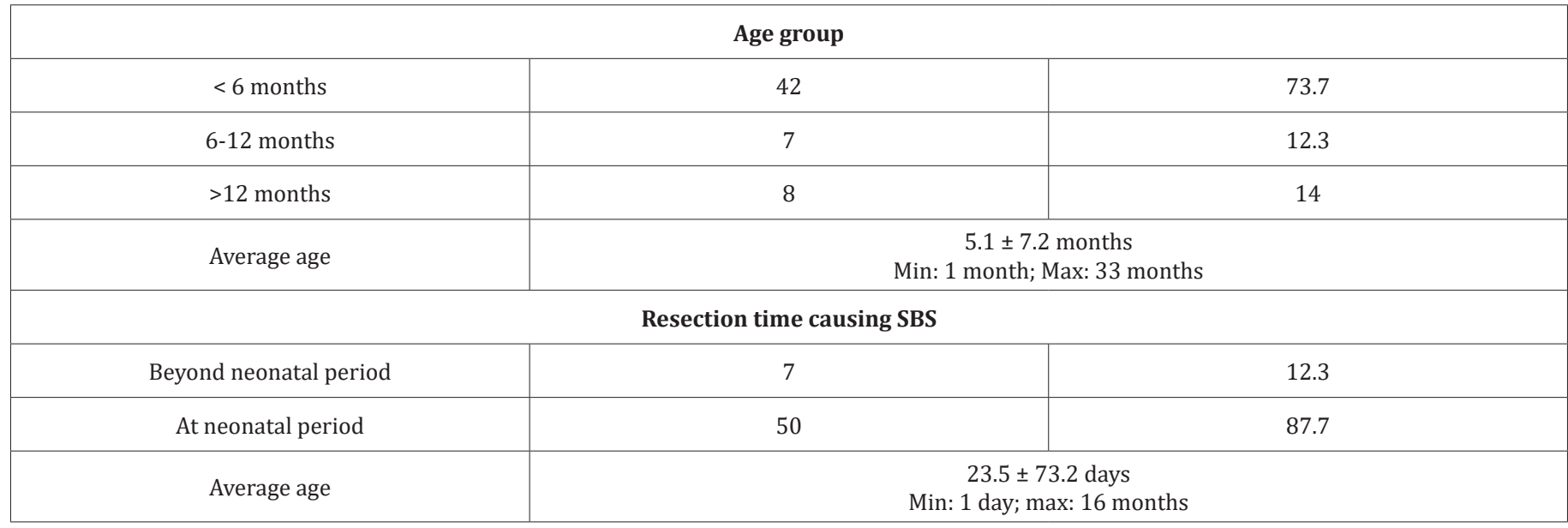

*Acquired SBS is classified by the Italian Federation of Societies of Digestive diseases and Italian Society of Pediatrics (2008).

${ }^{*}$ Disease phase: the disease has 3 phases. The acute phase ( $<3$ weeks post-operation) is characterized by TPN and recovery with massive loss of nutrients, water and electrolytes. Adaptation phase (weeks to months and usually 3 years) is characterized by poor intestinal absorption, reliant on PN. In phase 3(maintenance), nutrients are absorbed via GI tract $>70 \%$ demand and patients are not reliant on PN solutions.

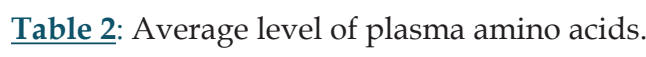

\begin{tabular}{|c|c|c|c|}
\hline Variables EAA & Average testing value $(\mu \mathrm{mol} / \mathrm{l})$ & Decrease n (\%) & Increase $\mathrm{N}(\%)$ \\
\hline Isoleucin & $53.1 \pm 27.9$ & 12.3 & 12.3 \\
\hline Leucin & $101,8 \pm 44.9$ & 0 & 10.5 \\
\hline Lysine & $148.1 \pm 62$ & 0 & 15.79 \\
\hline Methionine & $25.8 \pm 11.1$ & 3.5 & 10.5 \\
\hline Phenylalanine & $58.5 \pm 21.7$ & 1.8 & 12.3 \\
\hline Threonine & $127.1 \pm 73.9$ & 1.8 & 22.8 \\
\hline Tryptophan & $43.6 \pm 54.6$ & 19.3 & 5.3 \\
\hline Histidine & $49.4 \pm 21.3$ & 38.6 & 3.5 \\
\hline \multicolumn{4}{|c|}{ Non-essential amino acid (NEAA) } \\
\hline Alanie & $287.5 \pm 122.4$ & 10.5 & 8.8 \\
\hline Asparagine & $71.1 \pm 34.6$ & 0 & 21.1 \\
\hline Aspartate & $8.2 \pm 8.6$ & 14 & 5.3 \\
\hline Glutamate & $113.1 \pm 75.3$ & 0 & 28.1 \\
\hline Serine & $105.8 \pm 38.5$ & 15.8 & 3.5 \\
\hline Arginine & $36.5 \pm 27.2$ & 14 & 0 \\
\hline Cysteine & $27.6 \pm 18.9$ & 19.3 & 1.8 \\
\hline Glutamine & $745.5 \pm 412.8$ & 7 & 17.5 \\
\hline Tyrosine & $47.2 \pm 20$ & 8.8 & 0 \\
\hline Glycine & $186.4 \pm 105$ & 15.8 & 3.5 \\
\hline
\end{tabular}

Decrease, increase with $\mathrm{n}(\%)$ were the number and percentage of SBS children with AA concentrations changing vs. standard level of normal children at the same ages. 


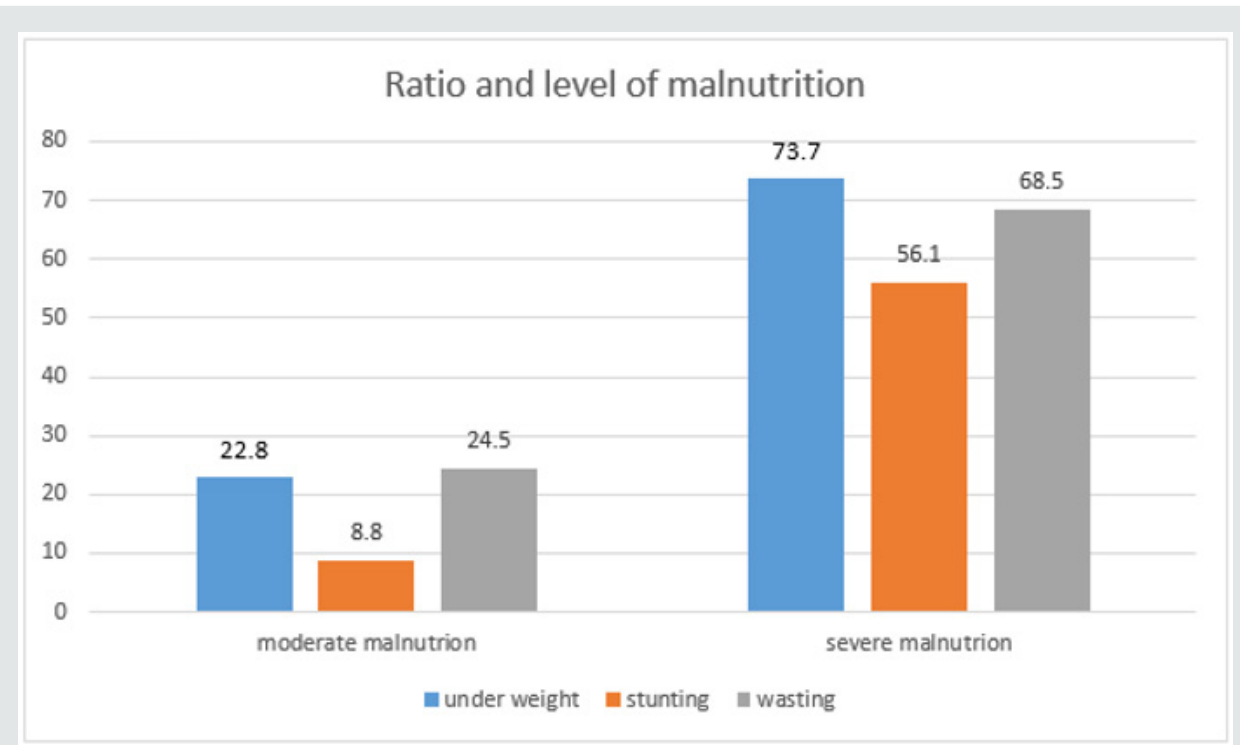

Graph 1: Malnutrition in children with SBS.

Malnutrition, $\mathrm{z}$-score ofW/A, W/H, H/A<-2SD is moderate malnutrition and from -2 to $\leq-3 S D$ is severe malnutrition. \% is the percentage of patients with nutritional status changing vs. the control.

The result (Graphs 2 \& 3) showed that average concentrations of EAA had no difference among phases and types of SBS, except threonine which was higher average value in adaptation phase than in maintenance phase and higher in functional SBS than in acquired SBS. The result (Table 3) showed that most patients had symptoms of dietary protein deficiency with $91.2 \%$ having decreasing plasma creatinine, $40.4 \%$ having decreasing plasma albumin and $38.6 \%$ having decreasing plasma protein. The result in Table 4 showed that
AA deficiency was more common in acquired SBS than in congenital SBS (77.4 vs. 57.7), in severe than in moderate underweight (73.8 vs. 61.5), in non-stunted children than in severe stunting (70 vs. 67.6), in non-infected children than in infected ones (91.7 vs. 62.2) with $p>0,05$. AA deficiency was less common in children in adaptation phase than in maintenance phase (59.5 vs. 93.3), in patients with artificial anal sphincter than in patients without artificial anal sphincter (61 vs. 87.5), with $\mathrm{p}<0.05$.

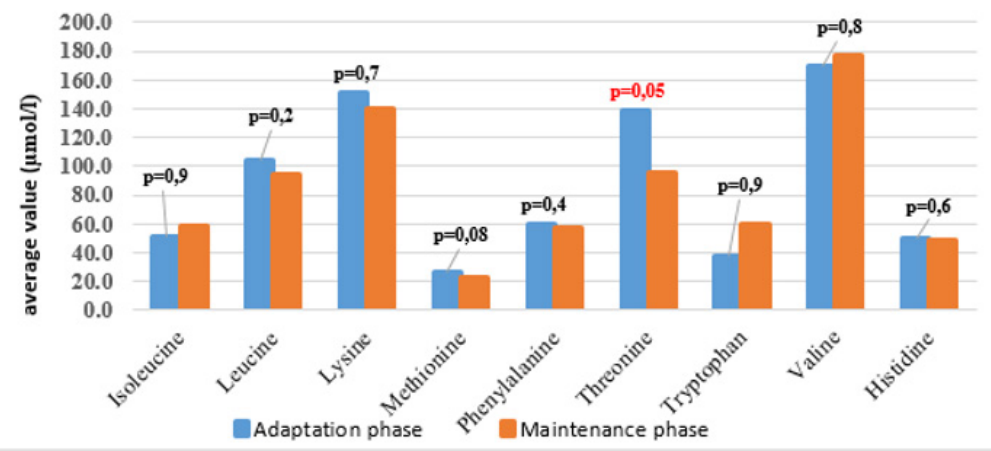

Graph 2: Average plasma concentration of EAA with each stages.

Table 3: General characteristics of children with SBS $(n=57)$.

\begin{tabular}{|c|c|c|c|}
\hline Variable & Average value & \% decrease & \% increase \\
\hline Urea $(\mathrm{mmol} / \mathrm{l})$ & $4.8 \pm 4$ & 17.5 & 12.3 \\
\hline Creatinine $(\mathrm{mmol} / \mathrm{l})$ & $37.7 \pm 13.8$ & 91.2 & 0 \\
\hline Albumin $(\mathrm{g} / \mathrm{l})$ & $36.4 \pm 6.2$ & 40.4 & 1.8 \\
\hline Protein $(\mathrm{g} / \mathrm{l})$ & $58.5 \pm 7.6$ & 38.6 & 0 \\
\hline
\end{tabular}

Some biochemical indices can be used to indirectly assess the status of AA and protein in the body.

Decrease. Increase: percentage of children with concentrations of biochemical substances decreasing/increase vs. normal level. 
Table 4: Deficiency of plasma AA and clinical status.

\begin{tabular}{|c|c|c|c|}
\hline \multirow{2}{*}{ Clinical Characteristics } & \multicolumn{2}{|c|}{ At least 1 AA deficiency } & \multirow{2}{*}{$\begin{array}{c}\text { p } \\
\text { (Fisher exact test) }\end{array}$} \\
\cline { 2 - 3 } & Yes (\%) & No (\%) & \multirow{2}{*}{0.02} \\
\hline Maintenance phase & 93.3 & 6.7 & \multirow{2}{*}{0.1} \\
\hline Adaptation phase & 59.5 & 40.5 & \multirow{2}{*}{0.05} \\
\hline Functional SBS & 57.7 & 22.6 & \\
\hline Acquired SBS & 77.4 & 12.5 & \multirow{2}{*}{0.4} \\
\hline Without artificial anal sphincter & 87.5 & 39 & \\
\hline With artificial anal sphincter & 61 & 38.5 & \\
\hline Moderate underweight & 61.5 & 30.2 & \multirow{2}{*}{0.85} \\
\hline Severe underweight & 73.8 & 32.4 & \\
\hline Non-stunted & 70 & 37.8 & \multirow{2}{*}{0.08} \\
\hline Stunted & 67.6 & 8.3 & \\
\hline With infection & 62.2 & & \\
\hline Without infection & 91.7 & & \\
\hline
\end{tabular}

At least 1 AA deficiency was percentage of SBS children who was in at least one AA deficiency

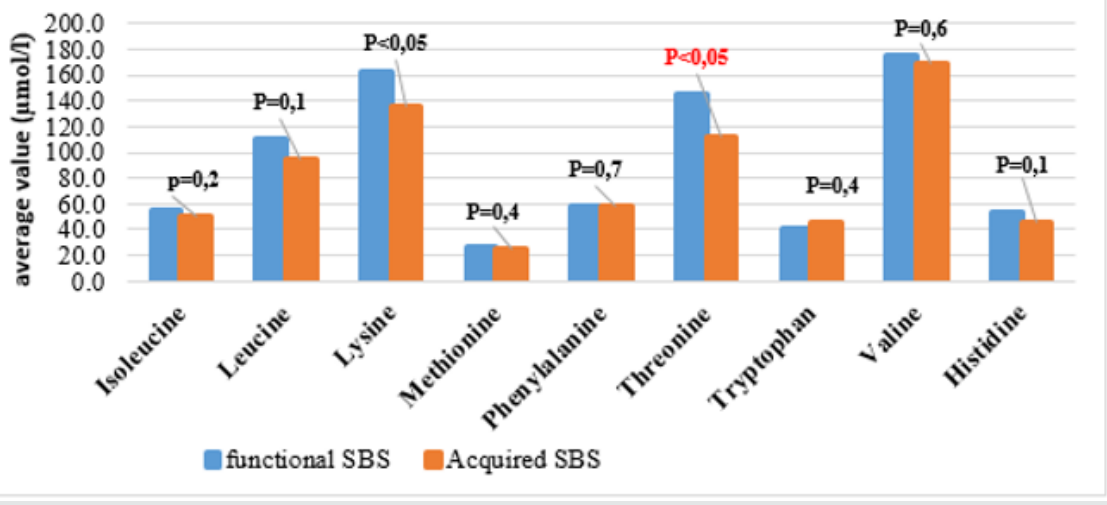

Graph 3: Average plasma concentration of EAA with types of SBS.

\section{Discussion}

The development of pediatric digestive surgery in recent years can solve many abdominal surgical diseases but along that, there is an increasing trend of SBS with early disease development due to the success of PN. The result (Table 1) showed that the ratio of patients under 6 months was $73.7 \%$ with average age of 5.1 months due to resection at neonatal stage (87.7\%) and average surgery age was23.5 days. The ratio of acquired SBS was54.4\%, functional SBSwas $45.6 \%, 73.7 \%$ patients were in adaptation phase and $26.3 \%$ were in maintenance phase. Besides, in Table 1, the ratio of young patients with artificial anal sphincter was71.9\%, so most patients did not have ileocecal valve; did not use colon for water, electrolyte and nutrient absorption and must be reliant on PN. The result (Graph 1) showed high ratio of malnutrition due to low age in the study (5.1 months) and early resection (23.5 days), which seriously affected patients' development and growth despite PN support. The study's limit was low number of patients, short study period so it was impossible to follow the disease progress but just evaluated at admission (cross-sectional study). In SBS, the integrity of intestinal functions were more important than appearance and the length of the remaining intestine. GI nutrition played an important role in increasing the adaptation of the remaining intestine and the results were reflected on changes of body weight and compositions. AA have many body functions so their redundancy or deficiency both cause negative impacts. The result (Table 2) showed that among 9 EAA (essential amino acid), differently from common assumption that most patients had decreasing EAA, all patients had higher average EAA concentrations than normal level with highest ratio of threonine $(22.8 \%)$ and lowest $(3.5 \%)$ of histidine. No patient had deficiency of leucine and lysine. Threonine has the function of mucin synthesis, which is essential for the maintenance of functions, gastric mucus integrity and immune functions. At present, threonine recommendation of WHO/FAO (2007) for male teenagers is $7 \mathrm{mg} / \mathrm{kg} /$ day [6] but must actually be $15 \mathrm{mg} / \mathrm{kg} /$ day to meet body demand [7]. 
We can evaluate threonine supply via leucine and/or phenylalanine balance, but leucine and phenylalanine (Table 2) both increased, so the oxidization of these $2 \mathrm{AA}$ and threonine using mechanism were ineffective, causing higher increase of threonine in patients with congenital SBS than in those with acquired SBS and in adaptation phase (much reliant on PN) than in maintenance phase (with oral intake) (Graphs $2 \& 3$ ). From the study result, EAA increase maybe unreal because the body did not use up these EAA for growth and recovery of injured gastric mucus. 38.6\% patients had histidine decrease. Histidine is the precursor for histamine (an important substance in allergic reaction), long-term histidine deficiency causes decreases in albumin (12\%), transferrin (17\%) and hemoglobin ( $\mathrm{Hb})(11 \%)$; increases secretion of urinary $\beta$-alanine, oxidation of phenylalanine [8]. The result (Graph 2) showed that $19.3 \%$ patients had tryptophan deficiency. Tryptophan is the precursor for serotonin (a neurotransmitter) so tryptophan deficiency causes depression and bad temper [9]. In our study, perhaps because patients were under 6 months, it was difficult to assess their personalities. We just documented their sleep disorder and mental growth retardation vs. normal children at the same ages and children with severe malnutrition. With NEAA (non-essential amino acid) (Table 2), all patients had NEAA increase including glutamate $(28.1 \%)$ and no patient had glutamate and asparagine decrease, glutamine increase (17.5\%). Glutamine is a prioritized fuel for intestinal and immune cells such as lymphocytes and macrophages. Although glutamine was not directly provided from nutritional feeds, it still had high plasma increase maybe because glutamine had been synthesized from glutamate and NH3, while there was glutamate in PN feeds with the concentration of $1.8 \mathrm{~g} / 250 \mathrm{ml}$ solution. Dietary glutamine supplementation increases gene expression of intestinal cell (120-124\%), which is necessary for cell growth, removal of oxidants, reducing (34-75\%) expression of genes that promote oxidative stress and activate immunity [10]. Dietary glutamine deficiency weakens cell signaling, leading to gut atrophy in both piglets and infants [11].

Glutamine and glutamate are main energy sources for small intestinal cells, depress protein digestion and catabolism, support gastric emptying and gastric motility, co-work with taurine and glycine to facilitate lipid digestion and absorption, regulate the growth and metabolism of micro flora in small intestine. Though there are many studies on glutamate oxidation in the gut [12], understanding on the efficacy of dietary glutamate supplementation on the integrity and functions of gastric mucus, especially under stress, and SBS conditions are still limited. In children with SBS, the gut integrity and length decrease, but the ratio of glutamine and glutamate increase is high, so the question is should we supplement glutamine for these patients and if yes, how should we supplement? Arginine improves the recovery of injured gastric mucus in pig, therefore arginine supplementation prevents necrotizing enterocolitis in premature infant especially those with poor activity of arginine synthesizing enzymes (internal arginine is metabolized from glutamate, glutamine and proline) or insufficiency of arginine [13]. $14 \%$ patients with SBS in the study had arginine decrease, $19.3 \%$ had cysteine deficiency while vaminolactused for feeding has $1 \mathrm{~g} / 1,000 \mathrm{ml}$ solution and cysteine is also metabolized from aspartate and NH3. Experiment on rats with SBS showed that dietary supplementation of cysteine and methionine can stimulate illeal mucosal growth and adaptation [14]. The supplementation of N-Acetyl - cysteine into PN solution with the dose $20-50 \mathrm{mg} /$ $\mathrm{kg} /$ day can alleviate elevated liver enzymes, increase plasma glutathione metabolism in children undergoing PN [15]. Therefore, in our study, we still saw a high ratio of patients with decreasing cysteine, which showed a limitation in treatment and the prognosis outcome of SBS patients. 15.8\% patients had serine decrease, while serine concentration in PN solution (vaminol act) is the highest among normal protein solutions $(3.8 \mathrm{~g} / 1,000 \mathrm{ml}$ solution).

From (Graphs 2 \& 3), it was seen that the average value of EAA in adaptation phase was higher than maintenance phase, in congenital SBS than in acquired SBS, however the difference is not significant except for threonine. AA concentrations of patients were higher in adaptation phase is because patients were provided not only oral but also PN feeds, they were not reliant on intestinal absorption, while patients in maintenance phase were just provided oral feeds so their plasma AA concentrations were totally reliant on intestinal absorption while the patients' GI tract could just absorb $70 \%$ of requirement. Commonly, children with serious illnesses or infections have increasing metabolism, catabolism and unbalanced nitrogen. Typical characteristic of catabolism is protein loss especially via urine and sweat (at high fever), increasing mobility of AA from muscles to circulation. AA is released from muscles especially glutamine and alanine, more than from synthesis. Normal children have more AA mobility than malnourished ones. To increase free AA synthesis, human body can use EAA from skeletal muscle, leading to EAA loss in skeletal muscle and wasting. There is a decrease of AA in the circulation and skeletal muscle. Generally, most AA decrease in the first stage of infection even before signs of fever. Glutamine has the most remarkable decrease. At serious illness; leucine, lysine, histidine and glutamine also decrease. On the contrary, phenylalanine and tryptophan have increasing plasma concentrations. Therefore, long-term illness like SBS shall cause wasting, muscle mass loss and lead to high rate of underweight (Graph 1) with 96.5\% underweight, 64.9\% stunting and $93 \%$ wasting. In this study, surprisingly, all patients received $\mathrm{AA}$ at a level higher than recommendation (AA in PN solution is $2 \mathrm{~g}$ / $\mathrm{kg}$ /dayand oral feed is nutramigen orpregestimil) but still had AA deficiency (Table 3) with $91.2 \%$ of decreasing plasma creatinine, decreasing plasma albumin (40.4\%) and decreasing plasma protein (38.6\%). This showed that intestinal AA absorption was low, maybe due to disorders of metabolism/AA synthesis or inefficient use of AA at serious illness, especially in adaptation phase.

In the study, there were up to $73.7 \%$ patients in adaptation phase, who were reliant on PN solutions. In this study, children 
under $5 \mathrm{~kg}$ used vaminolac as PN solution while children over $5 \mathrm{~kg}$ used amino plasma. Most patients in the study used vaminolactas they were under 2 years old and weighed under $5 \mathrm{~kg}$. Vaminolact has outstanding compositions of glutamic acid, leucine, alanine, lysine and with the presence of taurine, in contrast, has low level of histidine, isoleucine and tryptophan, therefore there was a high rate of patients with histidine, isoleucine, and tryptophan deficiencies. Though the reasons for the plasma concentration change of free AA are unknown, children with SBS have high rate of malnutrition, poor intestinal absorption, high growth demand, remarkably changing AA concentrations and this also reflects on their special requirement of AA. To ensure AA balance, dietary supplementation is necessary to compensate losses due to illnesses. The result (Table 4) showed that AA deficiencies in malnourished children, especially EAA. Richard (2016) studied on 313 children from 12 to 59 months with $62 \%$ of stunting. Stunted children had lower plasma concentration of EAA than non-stunted ones ( $p<0,01)$. Besides, stunted children had significant decrease of conditionally essential AA (arginine, glycine, glutamine), NEAA (asparagine, glutamate, serine) and 6 other sphingo lipids vs. non-stunted children [16].

\section{Conclusion}

High malnutrition rate in children patients with SBS, there were AA disorders in SBS children, patients' AA concentrations were highly reliant on protein compositions of PN solutions and intestinal absorption.

\section{Recommendation}

It is necessary to detect AA imbalance in SBS children, as well as carry out more further studies to have a recommendation on $\mathrm{AA}$ requirement of SBS children.

\section{References}

1. Suliman ME, Qureshi AR, Stenvinkel P (2005) Inflammation contributes to low plasma amino acid concentrations in patients with chronic kidney disease. Am J Clin Nutr 82(2): 342-349.

2. Dahlstrom KA, Ament ME, Laidlaw SA (1988) Plasma amino acid concentrations in children receiving long-term parenteral nutrition. J Pediatr Gastroenterol Nutr 7(5): 748-754.
3. Farida M ElBaz, Mona M Zaki, Azza M Youssef (2014) Study of plasma amino acid levels in children with autism: An Egyptian sample. Egyptian Journal of Medical Human Genetics 15(2): 181-186.

4. Wu G (2009) Amino acids: metabolism, functions, and nutrition Springer-Verlag 37(1): 1-17.

5. Xin Wu, Zheng Ruan, YunLing Gao (2010) Dietary supplementation with L-arginine or $\mathrm{N}$-carbamyl glutamate enhances intestinal growth and heat shock protein-70 expression in weanling pigs fed a corn- and soybean meal-based diet. Amino Acids 39(3): 831-839.

6. World Health Organ (2007) Protein and amino acid requirements in human nutrition. Tech Rep Ser (935): 1-265.

7. Anura V Kurpad, Tony Raj, Meredith M Regan (2002) Threonine requirements of healthy Indian men measured by a 24-h indicator amino acid oxidation and balance technique. The American Journal of Clinical Nutrition 76(4): 789-797.

8. Wantanee Kriengsinyos, Mahroukh Rafii, Linda J Wykes (2002) LongTerm Effects of Histidine Depletion on Whole-Body Protein Metabolism in Healthy Adults. J Nutr 132: 3340-3348.

9. Young SN (2013) The effect of raising and lowering tryptophan levels on human mood and social behavior. Phil Trans R Soc B 368(1615): 20110375.

10. Wang J, L Chen, P Li (2008). Gene expression is altered in piglet small intestine by weaning and dietary glutamine supplementation. J Nutr 138(6): 1025-1032.

11. Marc Rhoads J và WuG (2009) Glutamine, arginine, and leucine signaling in the intestine. Amino Acids 37(1): 111-122.

12. Stoll B và Burrin. DG (2006) Measuring splanchnic amino acid metabolism in vivo using maintenance isotopic tracers. J Anim Sci 84: 60-72.

13. Amin HJ, Zamora SA, Mc Millan DD (2002) Arginine supplementation prevents necrotizing enterocolitis in the premature infant. J Pediatr 140(4): 425-431.

14. Shyntum Y, Iyer SS, Tian J (2009) Dietary sulfur amino acid supplementation reduces small bowel thiol/disulfide redox state and stimulates ileal mucosal growth after massive small bowel resection in rats. J Nutr 139: 2272-2278.

15. Mager DR, Marcon M, Wales P, Pencharz PB (2008) Use of N-acetyl cysteine for the treatment of parenteral nutrition-induced liver disease in children receiving home parenteral nutrition. J Pediatr Gastroenterol Nutr 46: 220-223.

16. Richard D Semba, Michelle Shardell, Fayrouz A Sakr Ashour (2016) Child Stunting is Associated with Low Circulating Essential Amino Acids. E Bio Medicine 6: 246-252.

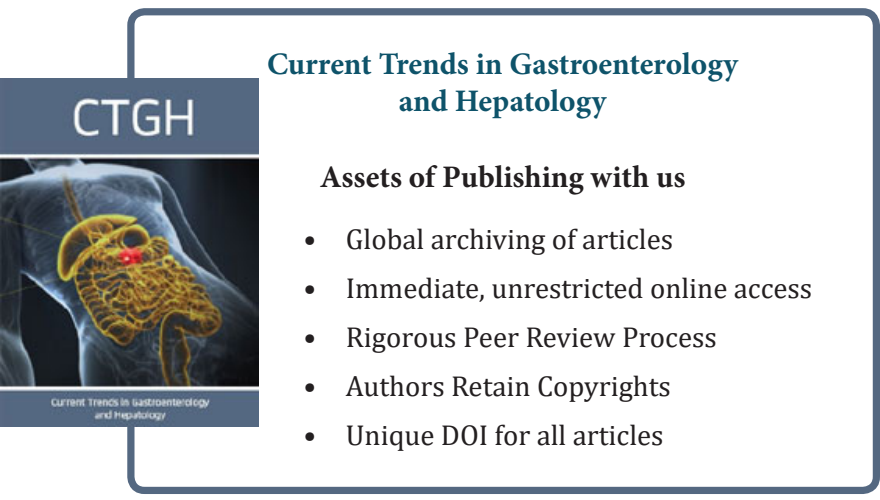

